\title{
Ultraviolet complete dark energy model
}

\author{
Gaurav Narain ${ }^{1, *}$ and Tianjun $\mathrm{Li}^{1,2, \dagger}$ \\ ${ }^{1}$ CAS Key Laboratory of Theoretical Physics, Institute of Theoretical Physics, \\ Chinese Academy of Sciences, Beijing 100190, China \\ ${ }^{2}$ School of Physical Sciences, University of Chinese Academy of Sciences, \\ No. 19A Yuquan Road, Beijing 100049, China
}

(Received 7 February 2018; published 30 April 2018)

\begin{abstract}
We consider a local phenomenological model to explain a nonlocal gravity scenario which has been proposed to address dark energy issues. This nonlocal gravity action has been seen to fit the data as well as $\Lambda$-CDM and therefore demands a more fundamental local treatment. The induced gravity model coupled with higher-derivative gravity is exploited for this proposal, as this perturbatively renormalizable model has a well-defined ultraviolet (UV) description where ghosts are evaded. We consider a generalized version of this model where we consider two coupled scalar fields and their nonminimal coupling with gravity. In this simple model, one of the scalar field acquires a vacuum expectation value (VEV), thereby inducing a mass for one of the scalar fields and generating Newton's constant. The induced mass however is seen to be always above the running energy scale thereby leading to its decoupling. The residual theory after decoupling becomes a platform for driving the accelerated expansion under certain conditions. Integrating out the residual scalar generates a nonlocal gravity action. The leading term of which is the nonlocal gravity action used to fit the data of dark energy.
\end{abstract}

DOI: 10.1103/PhysRevD.97.083523

\section{INTRODUCTION}

Dark energy (DE) has been a puzzling problem which is observed in the Universe in the era of large distance scale where the Universe is seen to be undergoing accelerated expansion $[1,2]$. There has been several efforts in order to explain at a fundamental level this observed phenomenon of accelerated expansion at large cosmic distances: quintessence [3-7], $\Lambda$-CDM, K-essence [8-10]. But so far $\Lambda$-CDM seems to have the best fit with the data. However, a good fundamental explanation is currently still lacking.

The idea of explaining accelerated expansion in the late time Universe with a cosmological-constant like term in the action goes back to the last century. However, the realization that such a term could play a role in explaining the observed accelerated expansion (as it give rise to negative pressure) is not very old. To explain the cause of this accelerated expansion which is obtained either by a constant energy-density term in the action or by some

\footnotetext{
gaunarain@itp.ac.cn

tti@itp.ac.cn
}

Published by the American Physical Society under the terms of the Creative Commons Attribution 4.0 International license. Further distribution of this work must maintain attribution to the author(s) and the published article's title, journal citation, and DOI. Funded by SCOAP ${ }^{3}$. field whose energy density asymptotically approaches a constant is known as dark energy.

A good and simple way to get an accelerated expansion is by making use of a scalar field where its slow variation with respect to cosmic time give rise to negative pressure. This is the usual scenario in the quintessence model [3-7] and k-essence model [8-10]. Of course, there are other procedures too which involves additional vector or bimetric gravity. Recently an interesting proposal using nonlocality has been suggested, as a way to get accelerated expansion [11-13]. It is seen that for a certain kind of nonlocality the accelerated expansion achieved in late-time Universe fits the data as nicely as $\Lambda$-CDM $[14,15]$. However so far the explanation for the appearance of this nonlocality does not exist, in the sense that it is not known whether it can arise from some fundamental local theories. Although it has been argued that similar kind of nonlocalities can arise in quantum theory where the energy dependence in the renormalization group running of couplings has been generalized such that $g(\mu) \rightarrow g(-\square)$, where $\square$ is the square of covariant derivative while $g$ is some coupling of the theory $[16,17]$. Here the infrared behavior of the running couplings is argued to lead to nonlocal modification of gravity. In this paper, we aim to address this issue of nonlocality by proposing a local model where such a nonlocality arises naturally when the fields decouple from the system. Such decoupling gives the nonlocal action which at late times results in accelerated expansion. 
The idea we exploit here is that in the higher-derivative induced gravity model, the quantum corrections give scalars an induced mass when scale-symmetry is broken via Coleman-Weinberg procedure [18]. However, the induced mass of scalar is seen to be always above the running energy scale resulting in a decoupling phenomenon [19-22]. In a model where there are more than one scalar, it is possible to achieve a phase of accelerated expansion at late times. In these cases if one of the scalar gets an induced mass after symmetry breaking and eventually decouples from the system, then the residual system can execute an era of accelerated expansion. We show that integrating out the extra scalar from the residual action results in a nonlocal action whose leading term matches with the nonlocal action considered by $[12,13,15]$.

The outline of paper is as follows. In Sec. II we give a brief review of the nonlocal gravity. In Sec. III we give a brief outline of induced and higher-derivative gravity. In Sec. IV we present the model which reduces to the nonlocal gravity model. Finally, the conclusion is presented in Sec. V.

\section{NONLOCAL GRAVITY}

Here in this section we will give a short review about the nonlocal gravity model which has been investigated in $[12,13,15]$. In this proposal the gravitational equation of motions are modified at long distances by a nonlocal term. This was first suggested in [23] where the general relativity (GR) equations were phenomenologically modified to

$$
\left(1-\mu^{2} \square^{-1}\right) G_{\mu \nu}=8 \pi G T_{\mu \nu} .
$$

Here $\mu^{2}$ dictates the characteristic length scale at which nonlocality enters, $G_{\mu \nu}$ is the Einstein tensor for the metric, $G$ is the gravitational coupling constant, and $T_{\mu \nu}$ is the energy-momentum tensor. However, an immediate problem that arises here is that the energy-momentum tensor is not automatically conserved as the covariant derivative $\nabla_{\mu}$ does not commute with $\square^{-1}$ on a curved space-time. It was soon realized that if one demands that energy-momentum tensor $T_{\mu \nu}$ should be covariantly conserved then one has to modify the equations of motions to

$$
G_{\mu \nu}-\mu^{2}\left(\square^{-1} G_{\mu \nu}\right)^{T}=8 \pi G T_{\mu \nu} .
$$

Here $T$ in the superscript denotes the transverse part of the tensor in brackets [24]. This modification implies that $T_{\mu \nu}$ is conserved but it leads to unstable cosmological evolution $[25,26]$. The first successful nonlocal model, free of instabilities was given by [25].

$$
G_{\mu \nu}-\frac{\mu^{2}}{3}\left(g_{\mu \nu} \square^{-1} R\right)^{T}=8 \pi G T_{\mu \nu},
$$

where the factor of $1 / 3$ was introduced to have a convenient normalization for the mass parameter. In this model, there is no Veltman-Zakharov discontinuity and it smoothly makes a transition to GR when $\mu^{2} \rightarrow 0$. Also, at cosmological scales, its evolution is stable during radiation and matter dominated era. At later times the nonlocal term behaves as dark energy giving rise to an accelerated expansion [25,26]. Moreover, its cosmological perturbations are well behaved both in scalar [27] and tensor sector [15,28]. Further investigations of this model reveals that it is consistent with $\mathrm{CMB}$, supernova, baryon acoustic oscillations (BAO), and structure formation data $[27,29,30]$. A detailed comparison with $\Lambda$-CDM shows that this nonlocal model fits the data at a level which is statistically indistinguishable from $\Lambda$-CDM [14,15]. This was named $R T$-model where $R$ stands for Ricci scalar while $T$ refers to the transverse part.

Currently an action for (3) does not exist. However, this model is closely related to following nonlocal action,

$$
S_{\mathrm{NL}}=\frac{M_{P}^{2}}{2} \int \mathrm{d}^{4} x \sqrt{-g}\left[R-\frac{\mu^{2}}{6} R \square^{-2} R\right],
$$

where $M_{P}$ is the reduced Planck mass. This action generates equation-of-motions (EOM) which on linearizing around flat space-time matches the one obtained after linearizing Eq. (3). However at the nonlinear level the two models are different. This model works very well at the background level [11] and matches nicely with data [15,27] (although it does not fit as well as $\Lambda$-CDM). This model is known as the $R R$-model (see [31] for its dynamical system analysis).

However, there are some arbitrariness in the sense of describing the nonlocal model given by Eq. (3) at the level of the action. This gives one freedom to consider various kinds of nonlocal actions. But the restrictions coming from stable cosmic evolution limit the number of allowed terms in the nonlocal modified action. It was shown that the following nonlinear extension

$$
S_{\mathrm{NL}}=\frac{M_{P}^{2}}{2} \int \mathrm{d}^{4} x \sqrt{-g}\left[R-\frac{\mu^{2}}{6} R(-\square+\xi R)^{-2} R\right],
$$

(where $\xi$ is a dimensionless parameter) does a very good job in matching the data. Such nonlinear extensions are inspired from the realization that the $R T$-model is a nonlinear extension of the $R R$-model [12]. This model has been studied extensively and can fit the DE data nicely (statistically as well as $\Lambda$-CDM).

\section{INDUCED GRAVITY MODEL}

Here we present a small review of the induced gravity model where we consider a nonminimally coupled scalar field with higher-derivative gravity action. The higher-derivative gravity action we consider is of the fourth order. The quantum theory of this is known to be renormalizable to all loops [32,33], and was recently 
shown to be unitary $[34,35]$ (see also references therein). This then offers a sufficiently simple quantum field theory of gravity which can be used to investigate physics at ultrahigh energies.

This scale-invariant model is like an induced gravity model [18], where a scalar acquires a VEV and in turn gives rise to the gravitational coupling as well as generating masses for other fields. The scale-invariant system consists of only dimensionless couplings. This makes the theory perturbatively renormalizable to all loops in fourdimensional space-time by power-counting [36,37] (for classical picture of these theories see [32,38]). Scaleinvariant gravitational systems coupled with matter have been investigated in the past. Some of the first studies were done in [36,37,39-41], where the renormalization group running of various couplings was computed and fixed point structure was analyzed. Further investigation for more complicated systems were done in [42-49] (see also the book [50] for more details).

Recently the topic has gained some momenta and these models have been reinvestigated [51-55]. The purpose of these papers was to see if it is possible to generate a scale dynamically starting from a scale-invariant system. In [51] the authors called their model "Agravity," where the Planck scale is dynamically generated from the VEV of a potential in the Einstein frame (not the Jordan frame). They achieve a negligible cosmological constant, generate the Planck's scale, and addresses naturalness [51,56] and inflation [57], but unitarity issues were not explored. ${ }^{1}$ In [18] it was realized that the induced mass of the ghost is always above the energy scale and hence is innocuous. In [52-55] the authors studied the issue of dynamical generation of scale via dimensional transmutation in the presence of background curvature. This also induces Einstein-Hilbert gravity and generates Newton's constant, but the unitarity problem was not addressed. An interesting idea has been suggested in $[59,60]$ by assuming an analogy with QCD, where the authors addressed the problem of ghosts and tachyons using the wisdom acquired from nonperturbative sector of QCD, as it is argued that the gravitational theory enters a nonperturbative regime below the Planck scale.

The idea of induced gravity goes back a long time. It was first proposed in $[61,62]$, where the quantum matter fluctuations at high energy generate gravitational dynamics at low energy inducing the cosmological and Newton's gravitational constant. Another proposal suggested in [63-65] induces Einstein gravity spontaneously via symmetry breaking along the lines of the Higgs mechanism. Later in [66-70] the idea of generation of Einstein gravity via dynamical symmetry breaking was considered, following

\footnotetext{
${ }^{1}$ In [58] a quantum mechanical treatment of 4-derivative theories was suggested, which when suitably extended can tackle more complicated field theoretic systems. This can perhaps address issues of ghosts and unitarity in a more robust manner.
}

the methodology of Coleman-Weinberg [71]. In [70], metric fluctuations were also incorporated in the generation of induced Newton's constant. Around the same time an induced gravity from Weyl-theory was studied [72-76] as well. Phase-transitions leading to the generation of EinsteinHilbert gravity due to loop-effects from a conformal factor coupled with a scalar field were studied in [77]. In [78,79] the renormalization group improved effective-potential of the dilaton leads to running of VEV thereby inducing mass scale (along with Einstein-Hilbert gravity). Furthermore, the authors make a proposal along lines of $[39,80]$ to tackle ghost and tachyons.

The renormalizable and UV well defined scale-invariant action that one considers here is

$$
\begin{aligned}
S_{\mathrm{GR}}= & \int \mathrm{d}^{4} x \sqrt{-g}\left[\frac{1}{16 \pi}\left\{-\frac{1}{f^{2}}\left(R_{\mu \nu} R^{\mu \nu}-\frac{1}{3} R^{2}\right)+\frac{\omega}{6 f^{2}} R^{2}\right\}\right. \\
& \left.+\frac{1}{2} \partial_{\mu} \phi \partial^{\mu} \phi-\frac{\lambda}{4} \phi^{4}-\frac{\xi}{2} R \phi^{2}\right],
\end{aligned}
$$

where the coupling parameters $f^{2}, \omega, \lambda$ and $\xi$ are all dimensionless, and the geometric quantities (curvature and covariant-derivative) depend on metric $g_{\mu \nu}$. If one decomposes the metric $g_{\mu \nu}=\bar{g}_{\mu \nu}+h_{\mu \nu}$, where $\bar{g}_{\mu \nu}$ is the background metric and $h_{\mu \nu}$ is the fluctuation, then one can compute the propagator of the fluctuation field $h_{\mu \nu}$ and its various couplings. If the background metric is flat then the quantum metric fluctuations in momentum space (for the Landau gauge condition $\partial^{\mu} h_{\mu \nu}=0$ ) is given by,

$D^{\mu \nu \rho \sigma}=\left(\Delta_{G}^{-1}\right)^{\mu \nu \rho \sigma}=(16 \pi) \frac{f^{2}}{q^{4}}\left(-2 P_{2}^{\mu \nu \rho \sigma}+\frac{1}{\omega} P_{s}^{\mu \nu \rho \sigma}\right)$,

where $P_{2}^{\mu \nu \rho \sigma}$ and $P_{s}^{\mu \nu \rho \sigma}$ are spin projectors. They can be written in flat space-time in momentum space in a simple form given by

$$
\begin{aligned}
& \left(P_{2}\right)_{\mu \nu}{ }^{\alpha \beta}=\frac{1}{2}\left[T_{\mu}{ }^{\alpha} T_{\nu}{ }^{\beta}+T_{\mu}{ }^{\beta} T_{\nu}{ }^{\alpha}\right]-\frac{1}{d-1} T_{\mu \nu} T^{\alpha \beta}, \\
& \left(P_{s}\right)_{\mu \nu}{ }^{\alpha \beta}=\frac{1}{d-1} T_{\mu \nu} T^{\alpha \beta},
\end{aligned}
$$

where

$$
L_{\mu \nu}=\frac{q_{\mu} q_{\nu}}{q^{2}}, \quad T_{\mu \nu}=\eta_{\mu \nu}-\frac{q_{\mu} q_{\nu}}{q^{2}} .
$$

These are basically the projector for projecting out various components of a vector field. They satisfy $q^{\mu} T_{\mu \nu}=0$ and $q^{\mu} L_{\mu \nu}=q_{\nu}$. Using them the projectors for the rank-2 tensor field can be constructed. The sign of couplings are taken in such a way so that the system does not generate any tachyons after the symmetry breaking [18]. This implies that $f^{2}>0, \omega>0, \lambda>0$, and $\xi>0$ (for the details on this 
choice of signs see $[18,81])$. For these choice of signs the system remains stable and tachyons free.

Due to quantum corrections a VEV is generated in the effective potential of the scalar field, which then becomes a new vacuum. The original $\varphi^{2}=0$ vacuum becomes unstable under quantum corrections and the field migrates to the new vacuum which occurs at $\varphi^{2}=\kappa^{2}$. It is given by [18]

$$
\left.\frac{\mathrm{d}}{\mathrm{d} \varphi^{2}} \operatorname{Re}\left(V_{\text {eff }}\right)\right|_{\varphi^{2}=\kappa^{2}}=0 .
$$

The generation of VEV consequently gives mass to scalar and generates an effective Newton's constant with the right sign, if the parameter $\xi$ was of opposite sign then the Newton's constant generated is of wrong sign [18]. The generated mass and Newton's coupling can be expressed in terms of VEV $\kappa^{2}$ and all the other couplings as

$$
m_{s}^{2}=\frac{3}{2} \lambda \kappa^{2}, \quad G^{-1}=8 \pi \xi \kappa^{2} .
$$

The graviton propagator after the symmetry breaking is following [18]

$$
\begin{aligned}
D^{\mu \nu, \alpha \beta}= & 16 \pi G \cdot\left[\frac{\left(2 P_{2}-P_{s}\right)^{\mu \nu, \alpha \beta}}{q^{2}+i \epsilon}+\frac{\left(P_{s}\right)^{\mu \nu, \alpha \beta}}{q^{2}-M^{2} / \omega+i \epsilon}\right. \\
& \left.-\frac{2\left(P_{2}\right)^{\mu \nu, \alpha \beta}}{q^{2}-M^{2}+i \epsilon}\right],
\end{aligned}
$$

where now $G$ is the induced Newton's constant and is defined using Eq. (12). The masses $M^{2}$ and $M^{2} / \omega$ are given by

$$
M^{2}=8 \pi f^{2} \cdot \xi \kappa^{2}, \quad \frac{M^{2}}{\omega}=8 \pi \frac{f^{2}}{\omega} \xi \kappa^{2} .
$$

From here we immediately note that if $\omega<0$ then there will be tachyons in the theory signaling an instability. The only way this catastrophe is avoided is when the parameter $\omega>0[18,34,35,81]$.

From the propagator in the broken phase (13) we realize that the last term has a wrong sign leading to trouble with unitarity. This is the consequence of having higherderivatives term in the action, resulting in ghosts. The generation of mass for the various modes allows one to investigate whether the induced mass for this ghost and its subsequent running can be such that it is always above the running energy scale. If the running of the parameters in the theory is such that the induced mass of the ghost remains always above the running energy scale, then the ghost mode never gets excited during the RG flow of the couplings. In the case of pure higher-derivative gravity without matter [34,35], it was indeed noticed from the RG running of parameters that there exists a large domain of coupling parameters where the ghost mass is always above the RG energy scale, and is innocuous. This phenomena was also witnessed in the case of induced gravity model investigated in [18]. The range of energy where the ghost remains physically unrealizable is quite large, extending from very high in the ultraviolet (at or more than Planck's scale) to very deep in infrared (almost cosmological scales). This range of energy is set by the parameter $\omega$ within which it remains positive and changes sign outside this range. For the system to be stable and tachyons free it is required that the parameter $\omega$ should remain positive $[34,35]$. The signs and stability of such higher-derivative systems have been discussed in more details in [81].

In the case of induced gravity [18], although the induced mass of the ghost is such that it is always above the energy scale, the occurrence of this phenomena leads to an interesting behavior for the scalar. The induced scalar mass is seen to be always above the energy scale leading to its decoupling in the same manner as for the higher-derivative ghost. It turns out that if one avoids ghosts to make the theory unitary then under appropriate choice of parameters, it is seen that the scalar gets also decoupled from the system. This decoupling of the scalar will have natural consequences in cosmology. In the following, we consider a generalization of this induced gravity action by incorporating an additional scalar field. One of the scalar gets a VEV and generates Newton's constant and masses, and gets decoupled. The leftover scalar in the residual action plays an interesting dynamics.

\section{MODEL}

In this section we present the idea of how the nonlocal action given in Eq. (5) can possibly arise from a local model via decoupling. This kind of nonlocal action can be safely embedded in the induced gravity scenario, which has an elegant UV completion [18]. The simple idea is that in the higher-derivative induced gravity action the scalar field gets completely decoupled from the system under certain circumstance when the ghosts are avoided. This decoupling can then be exploited to construct models which can give rise to nonlocalities in far infrared. As this model has a sensible UV completion, it becomes a good unified picture explaining some of the UV and IR physics together.

We consider the following two-scalar field model coupled nonminimally to gravity,

$$
\begin{aligned}
S= & \int \mathrm{d}^{4} x \sqrt{-g}\left[\frac{1}{2} \Phi^{T} \xi \Phi R+\frac{1}{2} \Phi^{T}(-\square) \Phi-V\left(\Phi^{T} \Phi\right)\right] \\
& +\int \mathrm{d}^{4} x \frac{\sqrt{-g}}{16 \pi}\left[-\frac{1}{f^{2}}\left(R_{\mu \nu} R^{\mu \nu}-\frac{1}{3} R^{2}\right)+\frac{\omega}{6 f^{2}} R^{2}\right],
\end{aligned}
$$

where $\Phi=\{\phi, \chi\}$ is a two real scalar field doublet. This model is an extension of the induced gravity model stated in Eq. (6), where the gravitational couplings are taken to 
have the same sign as before in order to ensure stability of system by no generating tachyons. The parameter $\xi$ is now a matrix whose entries are given by,

$$
\xi=\left(\begin{array}{cc}
\xi_{1} & \xi_{12} \\
\xi_{21} & \xi_{2}
\end{array}\right)
$$

where the entries of this matrix are all dimensionless. This is a simple two-scalar field model where the two scalar fields are not only coupled with each other but also have nonminimal coupling with gravity. It is a scale-invariant action which is renormalizable to all loops $[33,36,37] .^{2}$ As this model is just a minor extension (by inclusion of an additional scalar) of the induced gravity model stated in Eq. (6) where ghosts are evaded [18], so this model inherits the same virtues of the induced gravity model in which higher-derivative ghost are innocuous.

The potential is taken to be of $\phi^{4}$ type as in four dimensions it is the only allowed term which obeys scale symmetry and renormalizability (similar kind of models were also considered in $[82,83])$. The form of potential is given by

$$
V\left(\Phi^{T} \Phi\right)=\frac{1}{4}\left(\Phi^{T} \cdot \lambda \cdot \Phi\right)^{2}
$$

where the coupling $\lambda$ is a matrix

$$
\lambda=\left(\begin{array}{cc}
\lambda_{1} & \lambda_{12} \\
\lambda_{21} & \lambda_{2}
\end{array}\right)
$$

consisting of only dimensionless entries. In this model when the scalar $\phi$ acquires a VEV then it will generate mass terms for the various fields and will induce Newton's constant. Here we will not discuss the process through which VEV for $\phi$ gets generated but we assume that scale-symmetry breaking has already occurred resulting in a generation of nonzero VEV $\kappa$ for the scalar $\phi$. The paper [18] gives a detailed description of generation of VEV via a Coleman-Weinberg procedure, where the quantum fluctuations leads to symmetry breaking. The fluctuation of $\phi$ around the VEV are denoted by $\varphi$. Here in this paper we are interested in the aftermath of the symmetry breaking mechanism to know the behavior of this model at late times. The higher-derivatives gravity terms can be ignored as in the deep infrared they do not play a role and gets heavily suppressed [18]. This action can be written in an alternative form after opening up the matrix as follows

$$
\begin{aligned}
S= & \frac{1}{2} \int \mathrm{d}^{4} x \sqrt{-g}\left[\phi\left(-\square+\xi_{1} R\right) \phi+\chi\left(-\square+\xi_{2} R\right) \chi\right. \\
& +\phi\left(-2 \square+\xi_{12} R+\xi_{21} R\right) \chi \\
& \left.-\frac{1}{2}\left\{\lambda_{1} \phi^{2}+\left(\lambda_{12}+\lambda_{21}\right) \phi \chi+\lambda_{2} \chi^{2}\right\}^{2}\right] .
\end{aligned}
$$

If $\phi$-field acquires a VEV then it generates Newton's constant giving rise to the usual gravitational dynamics. However the fluctuation field $\varphi$ around the VEV of $\phi$ couples with the other scalar field $\chi$. The action after symmetry breaking is given by,

$$
\begin{aligned}
S= & \frac{1}{2} \int \mathrm{d}^{4} x \sqrt{-g}\left[\kappa^{2} \xi_{1} R+2 \kappa \xi_{1} \varphi R+\varphi\left(-\square+\xi_{1} R\right) \varphi\right. \\
& +\kappa\left(\xi_{12}+\xi_{21}\right) R \chi+\varphi\left(-2 \square+\xi_{12} R+\xi_{21} R\right) \chi \\
& +\chi\left(-\square+\xi_{2} R\right) \chi-\frac{1}{2}\left\{\lambda_{1} \kappa^{2}+2 \lambda_{1} \kappa \varphi+\left(\lambda_{12}+\lambda_{21}\right) \kappa \chi\right. \\
& \left.\left.+\lambda_{1} \varphi^{2}+\left(\lambda_{12}+\lambda_{21}\right) \varphi \chi+\lambda_{2} \chi^{2}\right\}^{2}\right] .
\end{aligned}
$$

The potential piece written in the second line when expanded will generate mass terms for the fields beside generating interactions. The mass term can be written in an elegant form in matrix notation as follows

$$
-\frac{1}{2}\left(\begin{array}{ll}
\varphi & \chi
\end{array}\right) \cdot \mathbb{M} \cdot\left(\begin{array}{l}
\varphi \\
\chi
\end{array}\right)=-\frac{1}{4} \kappa^{2}\left(\begin{array}{ll}
\varphi & \chi
\end{array}\right) \cdot\left(\begin{array}{cc}
6 \lambda_{1}^{2} & \lambda_{1}\left(\lambda_{12}+\lambda_{21}\right) \\
\lambda_{1}\left(\lambda_{12}+\lambda_{21}\right) & \left(\lambda_{12}+\lambda_{21}\right)^{2}+2 \lambda_{1} \lambda_{2}
\end{array}\right) \cdot\left(\begin{array}{l}
\varphi \\
\chi
\end{array}\right) .
$$

The interactions are given by

$$
\begin{aligned}
I= & -\frac{1}{4} \lambda_{1}^{2} \kappa^{4}-\frac{\lambda_{1} \kappa^{3}}{2}\left\{2 \lambda_{1} \varphi+\left(\lambda_{12}+\lambda_{21}\right) \chi\right\}-\frac{\kappa}{2}\left\{2 \lambda_{1}^{2} \varphi^{3}+3 \lambda_{1}\left(\lambda_{12}+\lambda_{21}\right) \chi \varphi^{2}+\left(\left(\lambda_{12}+\lambda_{21}\right)^{2}+2 \lambda_{1} \lambda_{2}\right) \varphi \chi^{2}+\left(\lambda_{12}+\lambda_{21}\right) \lambda_{2} \chi^{3}\right\} \\
& -\frac{1}{4}\left\{\lambda_{1}^{2} \varphi^{4}+\left(\left(\lambda_{12}+\lambda_{21}\right)^{2}+2 \lambda_{1} \lambda_{2}\right) \varphi^{2} \chi^{2}+2 \lambda_{1}\left(\lambda_{12}+\lambda_{21}\right) \varphi^{3} \chi+2 \lambda_{2}\left(\lambda_{12}+\lambda_{21}\right) \varphi \chi^{3}+\lambda_{2}^{2} \chi^{4}\right\} .
\end{aligned}
$$

\footnotetext{
${ }^{2}$ Higher-derivatives gravity terms ensures renormalizability of theory which remains unaffected by inclusion of renormalizable matter couplings.
} 
Here the first term is a like a vacuum energy term, the linear in fields will give rise to tadpoles in the quantum theory which can be absorbed via field redefinitions, the cubic and quartic interactions will give rise to nonlinear interactions. In special scenario where $\left(\lambda_{12}+\lambda_{21}\right)=0$, the mass matrix acquires a simple diagonal form while also simplifying the interaction pieces. We will consider this special case in the following. For this special case, the fields have the following masses (no mixing),

$$
m_{1}^{2}=3 \lambda_{1}^{2} \kappa^{2}, \quad m_{2}^{2}=\lambda_{1} \lambda_{2} \kappa^{2} .
$$

These are the induced masses for the fields $\varphi$ and $\chi$. The interaction piece for this special case is

$$
\begin{aligned}
I= & -\frac{1}{4} \lambda_{1}^{2} \kappa^{4}-\lambda_{1}^{2} \kappa^{3} \varphi-\lambda_{1} \kappa \varphi\left\{\lambda_{1} \varphi^{2}+\lambda_{2} \chi^{2}\right\} \\
& -\frac{1}{4}\left\{\lambda_{1}^{2} \varphi^{4}+2 \lambda_{1} \lambda_{2} \varphi^{2} \chi^{2}+\lambda_{2}^{2} \chi^{4}\right\} .
\end{aligned}
$$

One can then compute the equation of motion for the fields $\varphi$ (fluctuation) and $\chi$, respectively, by varying the full residual action as follows

$$
\begin{aligned}
(- & \left.+\xi_{1} R-m_{1}^{2}\right) \varphi+\left(\xi_{1} R-\lambda_{1} \kappa^{2}\right) \kappa \\
& +\frac{1}{2}\left(-2 \square+\xi_{12} R+\xi_{21} R\right) \chi-\lambda_{1} \kappa\left(3 \lambda_{1} \varphi^{2}+\lambda_{2} \chi^{2}\right) \\
& -\lambda_{1} \varphi\left(\lambda_{1} \varphi^{2}+\lambda_{2} \chi^{2}\right)=0, \\
(- & \left.+\xi_{2} R-m_{2}^{2}\right) \chi+\frac{1}{2}\left(\xi_{12}+\xi_{21}\right) \kappa R \\
& +\frac{1}{2}\left(-2 \square+\xi_{12} R+\xi_{21} R\right) \varphi-2 \lambda_{1} \lambda_{2} \kappa \varphi \chi \\
& -\lambda_{2} \chi\left(\lambda_{1} \varphi^{2}+\lambda_{2} \chi^{2}\right)=0,
\end{aligned}
$$

where each equation contains linear and nonlinear interactions terms. These are coupled differential equations. If the interaction strength is small (which is the case in cosmological scenarios) then one can solve the equations perturbatively. Moreover, in the scenario when the scalar $\varphi$ gets entirely decoupled from the system, the set of equations are greatly simplified. This is the approximation where mass $m_{1}^{2}$ is very large. In the induced gravity, higherderivative model considered in Eq. (6), such a phenomena naturally occurs when the scale-symmetry is broken. This breaking of scale symmetry not only induces the gravitational coupling (Newton's constant) but also induces mass for the various fields. Under the renormalization group running it is seen that the induced mass of the scalar is always above the running energy scale (under certain conditions [18]), resulting in a decoupling phenomena. This means that $m_{1}^{2} / E^{2}>1$ ( $E$ is the running energy). This condition means that the particle never goes on-shell. In infrared it is seen that this ratio $m_{1}^{2} / E^{2} \gg 1$, which will imply that $m_{1}^{2} \gg \square$. This also means that in quantum theories when such a particle appears inside a loop then it does not contribute to the imaginary part of the forward scattering amplitude (Cutkowsky cut), as it never goes on-shell. Here in this paper we will exploit this knowledge in our favor to decouple the scalar field fluctuation $\varphi$ from the system.

Under this decoupling approximation $m_{1}^{2} \gg \square$, the equation of motion for field $\varphi$ acquires a simplified expression. Keeping only the leading order terms we get

$$
\varphi=-\frac{1}{3} \kappa-\frac{1}{m_{1}^{2}}\left(\square \chi-\frac{\left(\xi_{12}+\xi_{21}\right) R \chi}{2}\right) .
$$

The other equation of motion for the field $\chi$ gets similarly simplified when the reduced equation of motion for $\varphi$ is plugged in it. This is given by,

$$
\left(-\square+\xi_{2} R-\frac{4}{9} m_{2}^{2}\right) \chi+\frac{\left(\xi_{12}+\xi_{21}\right) R}{3} \kappa=0,
$$

where higher order nonlinear terms are ignored. This residual linear equation can be solved easily by inverting the operator,

$$
\chi=-\frac{\kappa\left(\xi_{12}+\xi_{21}\right)}{3}\left(-\square+\xi_{2} R-\frac{4}{9} m_{2}^{2}\right)^{-1} R .
$$

This appears as a constraint in the system after decoupling of the scalar mode $\varphi$ has occurred. Here the inversion acts on the Ricci scalar $R$. One can then plug the solution for $\varphi$ from Eq. (27) back in the action of the theory, given in Eq. (20). This generates leading and subleading terms in the action. Under the decoupling approximation one can safely ignore the subleading part [which are of $\left.\mathrm{O}\left(1 / m_{1}^{2}\right)\right]$, as the dominant role will be played by leading part of the action. The leading part of the action is given by,

$$
\begin{aligned}
S= & \int \mathrm{d}^{4} x \sqrt{-g}\left[-\frac{4}{81} m_{1}^{2} \kappa^{2}+\frac{2 \xi_{1}}{9} \kappa^{2} R+\frac{\xi_{12}+\xi_{21}}{3} \kappa R \chi\right. \\
& \left.+\frac{1}{2}(\partial \chi)^{2}+\frac{\xi_{2}}{2} R \chi^{2}-\frac{2}{9} m_{2}^{2} \chi^{2}-\frac{1}{4} \lambda_{2}^{2} \chi^{4}\right] .
\end{aligned}
$$

This is the action where the decoupling of field $\varphi$ has occurred. This residual action contains only terms which are dominant after the decoupling (ignoring the contribution from $\left.\mathrm{O}\left(1 / m_{1}^{2}\right)\right)$. Here the field $\chi$ is coupled nonminimally with the background space-time. In the process of symmetry breaking a large cosmological constant is generated which remains present in the residual action for the field $\chi$. However, the effects of this large cosmological constant gets shielded if the gravitational coupling dictating the behavior of metric under the influence of cosmological constant, goes to zero. This situation actually occurs in the current case of induced gravity coupled with 
higher-derivatives (see Eq. (73), Fig. 7, Fig. 11 and Fig. 13 of [18]). Here it is noticed that renormalization group running of induced Newton's constant is such that it goes to zero in deep infrared. This behavior of gravitational Newton's constant was also witnessed in pure higherderivative gravity [34,35] (and in higher-derivative gravity coupled with gauge fields $[84,85])$. In then implies that in infrared as the gravitational coupling strength weakens, the backreaction of vacuum energy gets severely shielded making the large cosmological constant innocuous. In the following we will therefore ignore the generated cosmological constant term.

The field $\chi$ slow rolls for the case when coupling $\lambda_{2}$ and mass $m_{2}^{2}$ is small. In which case the kinetic term of the field $\chi$ can be ignored. This action has a simple form given by,

$$
\begin{aligned}
S= & \int \mathrm{d}^{4} x \sqrt{-g}\left[\frac{2 \xi_{1}}{9} \kappa^{2} R+\frac{\xi_{12}+\xi_{21}}{3} \kappa R \chi+\frac{\xi_{2}}{2} R \chi^{2}\right. \\
& \left.-\frac{2}{9} m_{2}^{2} \chi^{2}-\frac{1}{4} \lambda_{2}^{2} \chi^{4}\right] .
\end{aligned}
$$

In this slow-roll regime the field $\chi$ no longer has dynamics. It couples with the background curvature driving the dynamics of space-time. In this if the following approximation holds

$$
\frac{2 \xi_{1}}{3\left(\xi_{12}+\xi_{21}\right)} \gg \frac{\chi}{\kappa} \gg \frac{2\left(\xi_{12}+\xi_{21}\right)}{3 \xi_{2}}
$$

then the linear $\chi$ term does not contribute compared to quadratic and quartic pieces and the induced EinsteinHilbert piece.

The approximation in Eq. (32) is not unreasonable in the case of induced gravity coupled with higher-derivative gravity [18]. This model is a perturbatively renormalizable model in four dimensional space-time where the RG analysis does not put any constraint on the required values of the parameters $\xi_{1}, \xi_{2}, \xi_{12}$, and $\xi_{21}$. This means that these parameters can be chosen freely. Exploitation of this freedom into choosing those values of the parameters $\xi_{1}$, $\xi_{2}, \xi_{12}$, and $\xi_{21}$ such that the condition stated in Eq. (32) is fulfilled, suppresses the linear term in $\chi$ in the residual action stated Eq. (31). This will then leave us with the induced Einstein-Hilbert term, and quadratic terms in the fields plus the interaction pieces. This action is

$S=\int \mathrm{d}^{4} x \sqrt{-g}\left[\frac{2 \xi_{1}}{9} \kappa^{2} R+\frac{\xi_{2}}{2} R \chi^{2}-\frac{2}{9} m_{2}^{2} \chi^{2}-\frac{1}{4} \lambda_{2}^{2} \chi^{4}\right]$.

This action of the field $\chi$ is achieved once the decoupling of the heavy scalar has occurred, and the field $\chi$ starts to slow roll where it satisfies the condition Eq. (32). This results in a nondynamical action of $\chi$ field which couples with the background dynamical geometry. This action comes along with the constraint imposed in the Eq. (29). This constraint when plugged back into the residual action leads to the nonlocal action of the theory

$$
\begin{aligned}
S_{\mathrm{NL}}= & \int \mathrm{d}^{4} x \sqrt{-g}\left[\frac{2 \xi_{1}}{9} \kappa^{2} R-\frac{2 m_{2}^{2} \kappa^{2}\left(\xi_{12}+\xi_{21}\right)^{2}}{81}\right. \\
& \times R\left(-\square+\xi_{2} R-\frac{4}{9} m_{2}^{2}\right)^{-2} R+\frac{\xi_{2} \kappa^{2}\left(\xi_{12}+\xi_{21}\right)^{2}}{18} \\
& \times R\left(-\square+\xi_{2} R-\frac{4}{9} m_{2}^{2}\right)^{-1} R\left(-\square+\xi_{2} R-\frac{4}{9} m_{2}^{2}\right)^{-1} R \\
& \left.-\frac{\lambda_{2}^{2} \kappa^{4}}{324}\left(\xi_{12}+\xi_{21}\right)^{4}\left\{\left(-\square+\xi_{2} R-\frac{4}{9} m_{2}^{2}\right)^{-1} R\right\}^{4}\right] .
\end{aligned}
$$

Here the first term is the induced gravitational term while other terms are generated under decoupling and approximations. This can be seen as a heuristic derivation of the nonlocal action that has been studied extensively in $[12,13$, $15,28]$ and has been argued to reproduce dark energy data as good as $\Lambda$-CDM.

If we define the induced Newton's constant as $9 /(32 \pi G)^{-1}=\xi_{1} \kappa^{2}$, then one can pull out the factor of Planck's mass and write the action in a form which can be compared to the existing models given in $[12,13,15,28]$. This will become

$$
\begin{aligned}
S_{\mathrm{NL}}= & \frac{M_{p}^{2}}{2} \int \mathrm{d}^{4} x \sqrt{-g}\left[R-\frac{\mu^{2}}{6} R\left(-\square+\xi_{2} R-\frac{4}{9} m_{2}^{2}\right)^{-2} R\right. \\
& +\rho_{1}^{2} R\left(-\square+\xi_{2} R-\frac{4}{9} m_{2}^{2}\right)^{-1} \\
& \times R\left(-\square+\xi_{2} R-\frac{4}{9} m_{2}^{2}\right)^{-1} R \\
& \left.-\rho_{2}^{2}\left\{\left(-\square+\xi_{2} R-\frac{4}{9} m_{2}^{2}\right)^{-1} R\right\}^{4}\right]
\end{aligned}
$$

where $M_{P}^{2}=\left(4 \xi_{1} \kappa^{2}\right) / 9$ is the reduced Planck's mass and

$$
\begin{aligned}
\mu^{2} & =\frac{2\left(\xi_{12}+\xi_{21}\right)^{2} m_{2}^{2}}{3 \xi_{1}}, \\
\rho_{1}^{2} & =\frac{\xi_{2}\left(\xi_{12}+\xi_{21}\right)^{2}}{4 \xi_{1}}, \\
\rho_{2}^{2} & =\frac{\lambda_{2}^{2} M_{P}^{2}\left(\xi_{12}+\xi_{21}\right)^{4}}{32 \xi_{1}^{2}},
\end{aligned}
$$

where these entities are defined to make connection with the nonlocal model defined in Eq. (5). It was observed in $[12,13,15,28]$ that the parameter $\mu$ should be a very small 
quantity [of the $\mathcal{O}\left(H_{0}\right)$ ], in order to explain current accelerated expansion of the Universe. Following their work, it is seen one can still expect reasonable values of $m_{2}^{2}$ by appropriately choosing $\xi_{1}$ and $\left(\xi_{12}+\xi_{21}\right)$. The freedom allowed by the renormalizability of the theory allows one to choose these factors freely.

At this point one can make an order of estimate for the parameter $m_{2}^{2}$ by using the values of $\mu^{2}$ considered in $[12,13,15,28]$. From their analysis it is known that $\mu$ is of the order of current Hubble radius $H_{0}$. This is a very small quantity $\mathcal{O}\left(10^{-32}\right) \mathrm{eV}$. The authors of $[12,13,15,28]$ further mentions that even though $\mu$ (in their model) is a small quantity, but it does not describe the fundamental length scale that enters the physical system. This fundamental length scale is $\Lambda_{R R}$ and is related to $\mu^{2}$ via $M_{P}^{2}$ in the following manner,

$$
\Lambda_{R R}^{4}=\frac{M_{P}^{2}}{12} \mu^{2} .
$$

For our present case this will imply that $\Lambda_{R R}$ is related to the $m_{2}$ and $\kappa$ in the following way

$$
\Lambda_{R R}^{4}=\frac{2}{81}\left(\xi_{12}+\xi_{21}\right)^{2} m_{2}^{2} \kappa^{2} .
$$

According to the estimates made in works $[12,13,15,28]$, it is seen that for the reduced Plank mass $M_{P}$, if $\mu \sim$ $\mathcal{O}\left(H_{0}\right)$ then $\Lambda_{R R} \sim 10^{-3} \mathrm{eV}$. In GUT (grand unified theory models) scenarios, if the scale symmetry broke around the GUT scale resulting in generation of induced Newton's constant, then this will imply that $\kappa \sim 10^{16} \mathrm{GeV}$ and correspondingly $\xi_{1} \sim 100$. This knowledge further leads to the mass of the scalar field $\chi\left(m_{2}^{2}\right)$ following Eq. (38), which gives $m_{2} \sim 10^{-30}\left(\xi_{12}+\xi_{21}\right)^{-1} \mathrm{eV}$. Exploiting the freedom offered in the choice of values of parameters $\left(\xi_{12}+\xi_{21}\right)$, allows one to have a reasonable $m_{2}$. This particular model which has a well-defined UV completion and is free of ghosts can be seen to offer an interesting picture where low-energy nonlocal interaction emerges leading to accelerated expansion in late time Universe.

\section{CONCLUSION}

We tried to understand dark energy and the accelerated expansion it causes at late times of the Universe. We present a local unified model of a modified theory of gravity with a coupled system of scalar fields in Eq. (15). This scale-invariant model has a well-defined UV behavior in the sense that the theory is perturbatively renormalizable to all loops $[33,36,37]$ and in tachyons free regime higherderivative ghosts have been excised out $[18,34,35,39,80]$. In this model we would like to seek whether it can explain late time cosmic acceleration observed in the Universe. We considered a two coupled real scalar field model which also interacts nonminimally with gravity. One of the scalar acquires a VEV and in turn generates Newton's constant and masses for the fields. This scalar gets decoupled from the system as its mass becomes very large in the infrared, leaving behind a simple system.

Although breaking of scale-symmetry generates cosmological constant in the theory but its effect are shielded from affecting the dynamics of space-time due to the weakening of induced gravitational coupling constant in the infrared. This weakening of induced gravitational coupling is indeed observed in [18] (and for Einstein-Hilbert coupled with higher-derivatives in $[34,35,84,85])$, where such infrared vanishing of Newton's constant shield the effect of large cosmological constant. For the case of small coupling $\lambda_{2}$ and small mass, the field $\chi$ starts to slow roll, implying that its kinetic term can be ignored in the action. At this point the action for field $\chi$ consist of only nondynamical pieces and their interaction with the space-time. The RG running of the of parameters as seen in [18] is such that the approximation stated in Eq. (32) is satisfied if one chooses large values of $\xi_{1}$ and $\xi_{2}$. This does not hamper the renormalizability as explained in [18]. Under this condition the linear term in $\chi$ can be ignored over the quadratic and quartic piece. The integrating out of this leftover scalar from the simplified action leads to a nonlocal version of theory whose leading term matches the nonlocal gravity action studied extensively by [12-15,30] where it was noticed that it explain the late-time cosmic acceleration as well as $\Lambda$-CDM. This local scale-invariant system is then a good model which not only has a good UV completion but also leads to nonlocal gravity theory which can explain dark energy. In the papers [12-15,30] the authors introduced a controllable parameter $\mu^{2}$ (or $\Lambda_{R R}$ ) whose value was fixed by matching with the dark-energy data. However, an explanation for a possible origin of this length-scale was lacking. Here we derive the parameter $\mu^{2}$ (or $\Lambda_{R R}$ ) in terms of parameters present in the local model which is given in Eq. (38). This indicates a possible origin for length scale $\mu^{2}$ (or $\Lambda_{R R}$ ).

Generalized nonlocal gravity actions are also favorable in the sense that they offer superrenormalizability of the full theory, implying that the quantum theory is free of UV divergences [86-90]. The nonlocality present in these theories provides an extra suppression factor in the propagator at high-energies leading to a well defined UV behavior. Such nonlocal theories also arise at low energy from a more fundamental UV complete theory such as string theory. It will be interesting to see whether the model proposed in this paper can be elegantly extended to include these generalized nonlocal theories. This will be considered in a future publication.

The model presented here is UV complete (perturbatively renormalizable to all loops [33,36,37] where ghosts are evaded $[18,34,35,39,80]$ ) and approximations have been made in an attempt to arrive at the nonlocal form of the action. Perhaps an inclusion of additional symmetry 
incorporated at the level of local action might be able to give better handle over the scalar sector of the theory where the assumptions considered here will naturally arise. It may be possible that the residual scalar $\chi$ is a composite of some fundamental fermions, in which case dark energy arises when the condensate is formed [91]. This scenario will be explored in the future. Moreover, it will be interesting to investigate the modification of the behavior of infrared gravitational field theory (classical and quantum) under the inclusion of such nonlocalities. In particular the effect on gravitational waves (GW) created by such infrared nonlocal modification of gravity is worthy of investigation. Using future GW detectors one can possibly test such models [92,93]. This will be presented in future publication.

\section{ACKNOWLEDGMENTS}

We would like to thank Nirmalya Kajuri and Nick Houston for useful discussions during the course of this work. This research was supported by the Projects 11475238 and 11647601 supported by the National Natural Science Foundation of China, and by the Key Research Program of Frontier Science, CAS.
[1] A. G. Riess et al. (Supernova Search Team Collaboration), Observational evidence from supernovae for an accelerating universe and a cosmological constant, Astron. J. 116, 1009 (1998).

[2] S. Perlmutter et al. (Supernova Cosmology Project Collaboration), Measurements of Omega and Lambda from 42 high redshift supernovae, Astrophys. J. 517, 565 (1999).

[3] C. Wetterich, Cosmology and the fate of dilatation symmetry, Nucl. Phys. B302, 668 (1988).

[4] J. A. Frieman, C. T. Hill, A. Stebbins, and I. Waga, Cosmology with Ultralight Pseudo Nambu-Goldstone Bosons, Phys. Rev. Lett. 75, 2077 (1995).

[5] I. Zlatev, L. M. Wang, and P. J. Steinhardt, Quintessence, Cosmic Coincidence, and the Cosmological Constant, Phys. Rev. Lett. 82, 896 (1999).

[6] P. Brax and J. Martin, The robustness of quintessence, Phys. Rev. D 61, 103502 (2000).

[7] P. G. Ferreira and M. Joyce, Structure Formation with a Selftuning Scalar Field, Phys. Rev. Lett. 79, 4740 (1997).

[8] J. Garriga and V. F. Mukhanov, Perturbations in k-inflation, Phys. Lett. B 458, 219 (1999).

[9] C. Armendariz-Picon, T. Damour, and V. F. Mukhanov, k-inflation, Phys. Lett. B 458, 209 (1999).

[10] C. Armendariz-Picon, V. F. Mukhanov, and P. J. Steinhardt, A Dynamical Solution to the Problem of a Small Cosmological Constant and Late Time Cosmic Acceleration, Phys. Rev. Lett. 85, 4438 (2000).

[11] M. Maggiore and M. Mancarella, Nonlocal gravity and dark energy, Phys. Rev. D 90, 023005 (2014).

[12] G. Cusin, S. Foffa, M. Maggiore, and M. Mancarella, Conformal symmetry and nonlinear extensions of nonlocal gravity, Phys. Rev. D 93, 083008 (2016).

[13] M. Maggiore, Nonlocal infrared modifications of gravity. A review, Fundam. Theor. Phys. 187, 221 (2017).

[14] Y. Dirian, S. Foffa, M. Kunz, M. Maggiore, and V. Pettorino, Non-local gravity and comparison with observational datasets, J. Cosmol. Astropart. Phys. 04 (2015) 044.

[15] Y. Dirian, S. Foffa, M. Kunz, M. Maggiore, and V. Pettorino, Non-local gravity and comparison with observational datasets. II. Updated results and Bayesian model comparison with $\Lambda \mathrm{CDM}, \mathrm{J}$. Cosmol. Astropart. Phys. 05 (2016) 068 .

[16] M. Maggiore, Dark energy and dimensional transmutation in $R^{2}$ gravity, arXiv:1506.06217.

[17] M. Maggiore, Perturbative loop corrections and nonlocal gravity, Phys. Rev. D 93, 063008 (2016).

[18] G. Narain, Exorcising ghosts in induced gravity, Eur. Phys. J. C 77, 683 (2017).

[19] T. Appelquist and J. Carazzone, Infrared singularities and massive fields, Phys. Rev. D 11, 2856 (1975).

[20] E. V. Gorbar and I. L. Shapiro, Renormalization group and decoupling in curved space, J. High Energy Phys. 02 (2003) 021.

[21] E. V. Gorbar and I. L. Shapiro, Renormalization group and decoupling in curved space. 2. The standard model and beyond, J. High Energy Phys. 06 (2003) 004.

[22] E. V. Gorbar and I. L. Shapiro, Renormalization group and decoupling in curved space. 3. The case of spontaneous symmetry breaking, J. High Energy Phys. 02 (2004) 060.

[23] N. Arkani-Hamed, S. Dimopoulos, G. Dvali, and G. Gabadadze, Nonlocal modification of gravity and the cosmological constant problem, arXiv:hep-th/0209227.

[24] M. Jaccard, M. Maggiore, and E. Mitsou, Nonlocal theory of massive gravity, Phys. Rev. D 88, 044033 (2013).

[25] M. Maggiore, Phantom dark energy from nonlocal infrared modifications of general relativity, Phys. Rev. D 89, 043008 (2014).

[26] S. Foffa, M. Maggiore, and E. Mitsou, Cosmological dynamics and dark energy from nonlocal infrared modifications of gravity, Int. J. Mod. Phys. A 29, 1450116 (2014).

[27] Y. Dirian, S. Foffa, N. Khosravi, M. Kunz, and M. Maggiore, Cosmological perturbations and structure formation in nonlocal infrared modifications of general relativity, J. Cosmol. Astropart. Phys. 06 (2014) 033.

[28] G. Cusin, S. Foffa, M. Maggiore, and M. Mancarella, Nonlocal gravity with a Weyl-square term, Phys. Rev. D 93, 043006 (2016).

[29] S. Nesseris and S. Tsujikawa, Cosmological perturbations and observational constraints on nonlocal massive gravity, Phys. Rev. D 90, 024070 (2014). 
[30] A. Barreira, B. Li, W. A. Hellwing, C. M. Baugh, and S. Pascoli, Nonlinear structure formation in nonlocal gravity, J. Cosmol. Astropart. Phys. 09 (2014) 031.

[31] H. Nersisyan, Y. Akrami, L. Amendola, T. S. Koivisto, and J. Rubio, Dynamical analysis of $R \frac{1}{\square^{2}} R$ cosmology: Impact of initial conditions and constraints from supernovae, Phys. Rev. D 94, 043531 (2016).

[32] K. S. Stelle, Classical gravity with higher derivatives, Gen. Relativ. Gravit. 9, 353 (1978).

[33] K. S. Stelle, Renormalization of higher derivative quantum gravity, Phys. Rev. D 16, 953 (1977).

[34] G. Narain and R. Anishetty, Short distance freedom of quantum gravity, Phys. Lett. B 711, 128 (2012).

[35] G. Narain and R. Anishetty, Unitary and renormalizable theory of higher derivative gravity, J. Phys. Conf. Ser. 405, 012024 (2012).

[36] E. S. Fradkin and A. A. Tseytlin, Renormalizable asymptotically free quantum theory of gravity, Phys. Lett. B 104B, 377 (1981).

[37] E. S. Fradkin and A. A. Tseytlin, Renormalizable asymptotically free quantum theory of gravity, Nucl. Phys. B201, 469 (1982).

[38] L. Alvarez-Gaume, A. Kehagias, C. Kounnas, D. Lust, and A. Riotto, Aspects of quadratic gravity, Fortsch. Phys. 64, 176 (2016).

[39] J. Julve and M. Tonin, Quantum gravity with higher derivative terms, Nuovo Cimento Soc. Ital. Fis. 46, 137 (1978).

[40] N. H. Barth and S. M. Christensen, Quantizing fourth order gravity theories. 1. The functional integral, Phys. Rev. D 28, 1876 (1983).

[41] I. G. Avramidi and A. O. Barvinsky, Asymptotic freedom in higher derivative quantum gravity, Phys. Lett. B 159B, 269 (1985).

[42] I. L. Buchbinder, O. K. Kalashnikov, I. L. Shapiro, V. B. Vologodsky, and J. J. Wolfengaut, The stability of asymptotic freedom in grand unified models coupled to $R^{2}$ gravity, Phys. Lett. B 216, 127 (1989).

[43] I. L. Shapiro, Asymptotic behavior of effective Yukawa coupling constants in quantum $R^{2}$ gravity with matter, Classical Quantum Gravity 6, 1197 (1989).

[44] S. D. Odintsov, The parametrization invariant and gauge invariant effective actions in quantum field theory, Fortsch. Phys. 38, 371 (1990).

[45] E. Elizalde, S. D. Odintsov, and A. Romeo, Improved effective potential in curved space-time and quantum matter, higher derivative gravity theory, Phys. Rev. D 51, 1680 (1995).

[46] E. Elizalde, C. O. Lousto, S. D. Odintsov, and A. Romeo, GUTs in curved space-time: Running gravitational constants, Newtonian potential and the quantum corrected gravitational equations, Phys. Rev. D 52, 2202 (1995).

[47] E. Elizalde, S. D. Odintsov, and A. Romeo, Manifestations of quantum gravity in scalar QED phenomena, Phys. Rev. D 51, 4250 (1995).

[48] G. de Berredo-Peixoto and I. L. Shapiro, Conformal quantum gravity with the Gauss-Bonnet term, Phys. Rev. D 70, 044024 (2004).

[49] G. de Berredo-Peixoto and I. L. Shapiro, Higher derivative quantum gravity with Gauss-Bonnet term, Phys. Rev. D 71, 064005 (2005).
[50] I. L. Buchbinder, S. D. Odintsov, and I. L. Shapiro, Effective Action in Quantum Gravity (IOP, Bristol, 1992), p. 413.

[51] A. Salvio and A. Strumia, Agravity, J. High Energy Phys. 06 (2014) 080.

[52] M. B. Einhorn and D. R. T. Jones, Naturalness and dimensional transmutation in classically scale-invariant gravity, J. High Energy Phys. 03 (2015) 047.

[53] T. Jones and M. Einhorn, Quantum gravity and dimensional transmutation, Proc. Sci. PLANCK (2015) 061.

[54] M. B. Einhorn and D. R. T. Jones, Induced gravity I: Real scalar field, J. High Energy Phys. 01 (2016) 019.

[55] M. B. Einhorn and D. R. T. Jones, Induced gravity II: Grand unification, J. High Energy Phys. 05 (2016) 185.

[56] A. Salvio, Solving the standard model problems in softened gravity, Phys. Rev. D 94, 096007 (2016).

[57] K. Kannike, G. Htsi, L. Pizza, A. Racioppi, M. Raidal, A. Salvio, and A. Strumia, Dynamically induced Planck scale and inflation, J. High Energy Phys. 06 (2015) 065.

[58] A. Salvio and A. Strumia, Quantum mechanics of 4-derivative theories, Eur. Phys. J. C 76, 227 (2016).

[59] B. Holdom and J. Ren, QCD analogy for quantum gravity, Phys. Rev. D 93, 124030 (2016).

[60] B. Holdom and J. Ren, Quadratic gravity: From weak to strong, Int. J. Mod. Phys. D 25, 1643004 (2016).

[61] Y. B. Zeldovich, Cosmological constant and elementary particles, Pis'ma Zh. Eksp. Teor. Fiz. 6, 883 (1967) [JETP Lett. 6, 316 (1967)].

[62] A. D. Sakharov, Vacuum quantum fluctuations in curved space and the theory of gravitation, Dokl. Akad. Nauk Ser. Fiz. 177, 70 (1967) [Sov. Phys. Dokl. 12, 1040 (1968)]; Sov. Phys. Usp. 34, 394 (1991); Gen. Relativ. Gravit. 32, 365 (2000).

[63] Y. Fujii, Scalar-tensor theory of gravitation and spontaneous breakdown of scale invariance, Phys. Rev. D 9, 874 (1974).

[64] E. M. Chudnovsky, The spontaneous conformal symmetry breaking and Higgs model, Teor. Mat. Fiz. 35, 398 (1978) [Theor. Math. Phys. 35, 538 (1978)].

[65] A. Zee, A Broken Symmetric Theory of Gravity, Phys. Rev. Lett. 42, 417 (1979).

[66] S. L. Adler, A formula for the induced gravitational constant, Phys. Lett. B 95, 241 (1980).

[67] S. L. Adler, Order R Vacuum Action Functional in Scalar Free Unified Theories with Spontaneous Scale Breaking, Phys. Rev. Lett. 44, 1567 (1980).

[68] S. L. Adler, Induced gravitation, AIP Conf. Proc. 68, 915 (1980).

[69] A. Zee, Spontaneously generated gravity, Phys. Rev. D 23, 858 (1981).

[70] S. L. Adler, Einstein gravity as a symmetry-breaking effect in quantum field theory, Rev. Mod. Phys. 54, 729 (1982); Erratum, 55, 837 (1983).

[71] S. R. Coleman and E. J. Weinberg, Radiative corrections as the origin of spontaneous symmetry breaking, Phys. Rev. D 7, 1888 (1973).

[72] R. I. Nepomechie, Einstein gravity as the low-energy effective theory of Weyl gravity, Phys. Lett. 136B, 33 (1984).

[73] A. Zee, Einstein gravity emerging from quantum Weyl gravity, Ann. Phys. (N.Y.) 151, 431 (1983).

[74] I. L. Buchbinder, Mechanism for induction of Einstein gravitation, Sov. Phys. J. 29, 220 (1986). 
[75] E. Elizalde and S. D. Odintsov, Gravitational phase transitions in infrared quantum gravity, Mod. Phys. Lett. A 08, 3325 (1993).

[76] S. D. Odintsov and I. L. Shapiro, General relativity as the low-energy limit in higher derivative quantum gravity, Classical Quantum Gravity 9, 873 (1992); , Curvature phase transition in $R^{2}$ gravity and induction of Einstein gravity, Teor. Mat. Fiz. 90, 469 (1992) [Theor. Math. Phys. 90, 319 (1992)].

[77] I. L. Shapiro and G. Cognola, Interaction of low-energy induced gravity with quantized matter and phase transition induced to curvature, Phys. Rev. D 51, 2775 (1995).

[78] R. Floreanini and R. Percacci, Average effective potential for the conformal factor, Nucl. Phys. B436, 141 (1995).

[79] R. Floreanini and R. Percacci, The renormalization group flow of the Dilaton potential, Phys. Rev. D 52, 896 (1995).

[80] A. Salam and J. A. Strathdee, Remarks on high-energy stability and renormalizability of gravity theory, Phys. Rev. D 18, 4480 (1978).

[81] G. Narain, Signs and stability in higher-derivative gravity, Int. J. Mod. Phys. A 33, 1850031 (2018).

[82] J. Garcia-Bellido, J. Rubio, M. Shaposhnikov, and D. Zenhausern, Higgs-dilaton cosmology: From the early to the late universe, Phys. Rev. D 84, 123504 (2011).

[83] G. K. Karananas and J. Rubio, On the geometrical interpretation of scale-invariant models of inflation, Phys. Lett. B 761, 223 (2016).
[84] G. Narain and R. Anishetty, Charge renormalization due to graviton loops, J. High Energy Phys. 07 (2013) 106.

[85] G. Narain and R. Anishetty, Running couplings in quantum theory of gravity coupled with gauge fields, J. High Energy Phys. 10 (2013) 203.

[86] J. W. Moffat, Ultraviolet complete quantum gravity, Eur. Phys. J. Plus 126, 43 (2011).

[87] T. Biswas, T. Koivisto, and A. Mazumdar, Towards a resolution of the cosmological singularity in non-local higher derivative theories of gravity, J. Cosmol. Astropart. Phys. 11 (2010) 008.

[88] L. Modesto, Super-renormalizable quantum gravity, Phys. Rev. D 86, 044005 (2012).

[89] F. Briscese, L. Modesto, and S. Tsujikawa, Superrenormalizable or finite completion of the Starobinsky theory, Phys. Rev. D 89, 024029 (2014).

[90] L. Modesto and L. Rachwal, Super-renormalizable and finite gravitational theories, Nucl. Phys. B889, 228 (2014).

[91] J. R. Bhatt, B. R. Desai, E. Ma, G. Rajasekaran, and U. Sarkar, Neutrino condensate as origin of dark energy, Phys. Lett. B 687, 75 (2010).

[92] E. Belgacem, Y. Dirian, S. Foffa, and M. Maggiore, Nonlocal gravity. Conceptual aspects and cosmological predictions, J. Cosmol. Astropart. Phys. 03 (2018) 002.

[93] E. Belgacem, Y. Dirian, S. Foffa, and M. Maggiore, The gravitational-wave luminosity distance in modified gravity theories, arXiv:1712.08108. 